\title{
What Are the Chances?
}

The approach to clinical conundrums by an expert clinician is revealed through the presentation of an actual patient's case in an approach typical of a morning report. Similarly to patient care, sequential pieces of information are provided to the clinician, who is unfamiliar with the case. The focus is on the thought processes of both the clinical team caring for the patient and the discussant.

This icon represents the patient's case. Each paragraph that follows represents the discussant's thoughts.

Stefanie Deeds, $M D^{1}$, Patricia Oakes, $\mathrm{MD}^{2}$, Somnath Mookherjee, $M D^{1}$, David S. Levitt, $\mathrm{MD}^{1 *}$

'Department of Medicine and '2Department of Neurology, University of Washington School of Medicine, Seattle, Washington.

Two weeks after undergoing a below-knee amputation

(BKA) and 10 days after being discharged to a skilled nursing facility (SNF), an 87-year-old man returned to the emergency department (ED) for evaluation of somnolence and altered mental state. In the ED, he was disoriented and unable to provide a detailed history.

The differential diagnosis for acute confusion and altered consciousness is broad. Initial possibilities include toxic-metabolic abnormalities, medication side effects, and infections. Urinary tract infection, pneumonia, and surgical-site infection should be assessed for first, as they are common causes of postoperative altered mentation. Next to be considered are subclinical seizure, ischemic stroke, and infectious encephalitis or meningitis, along with hemorrhagic stroke and subdural hematoma.

During initial assessment, the clinician should ascertain baseline mental state, the timeline of the change in mental status, recent medication changes, history of substance abuse, and concern about any recent trauma, such as a fall. Performing the physical examination, the clinician should assess vital signs and then focus on identifying localizing neurologic deficits.

First steps in the work-up include a complete metabolic panel, complete blood cell count, urinalysis with culture, and a urine toxicology screen. If the patient has a "toxic" appearance, blood cultures should be obtained. An electrocardiogram should be used to screen for drug toxicity or evidence of cardiac ischemia. If laboratory test results do not reveal an obvious infectious or metabolic cause, a noncontrast computed tomography (CT) of the head should be obtained. In terms of early interventions, a low glucose level should be treated with thiamine and then glucose, and naloxone should be given if there is any suspicion of narcotic overdose.

\footnotetext{
*Address for correspondence and reprint requests: David Levitt, MD, Department of Medicine, University of Washington School of Medicine, 325 Ninth Ave, Campus Box 359780, Seattle, WA 98104; Telephone: 206-744-7791; Fax: 888-847-9518; E-mail: figment@uw.edu
}

Received: March 31, 2016; Revised: September 2, 2016; Accepted: September 18, 2016

2017 Society of Hospital Medicine DOI 10.12788/jhm.2718
More history was obtained from the patient's records. The BKA was performed to address a nonhealing transmetatarsal amputation. Two months earlier, the transmetatarsal amputation had been performed as treatment for a diabetic forefoot ulcer with chronic osteomyelitis. The patient's post-BKA course was uncomplicated. $\mathrm{He}$ was started on intravenous (IV) ertapenem on postoperative day 1 , and on postoperative day 4 was discharged to the SNF to complete a 6-week course of antibiotics for osteomyelitis. Past medical history included paroxysmal atrial fibrillation, coronary artery disease, congestive heart failure (ejection fraction 40\%), and type 2 diabetes mellitus. Medications given at the SNF were oxycodone, acetaminophen, cholecalciferol, melatonin, digoxin, ondansetron, furosemide, gabapentin, correctional insulin, tamsulosin, senna, docusate, warfarin, and metoprolol. While there, the patient's family expressed concern about his diminishing "mental ability." They reported he had been fully alert and oriented on arrival at the SNF, and living independently with his wife before the BKA. Then, a week before the ED presentation, he started becoming more somnolent and forgetful. The gabapentin and oxycodone dosages were reduced to minimize their sedative effects, but he showed no improvement. At the SNF, a somnolence work-up was not performed.

Several of the patient's medications can contribute to altered mental state. Ertapenem can cause seizures as well as profound mental status changes, though these are more likely in the setting of poor renal function. The mental status changes were noticed about a week into the patient's course of antibiotics, which suggests a possible temporal correlation with the initiation of ertapenem. An electroencephalogram is required to diagnose nonconvulsive seizure activity. Narcotic overdose should still be considered, despite the recent reduction in oxycodone dosage. Digoxin toxicity, though less likely when the dose is stable and there are no changes in renal function, can cause a confused state. Concurrent use of furosemide could potentiate the toxic effects of digoxin.

Non-medication-related concerns include hypoglycemia, hyperglycemia, and, given his history of atrial fibrillation, cardioembolic stroke. Although generalized confusion is 
not a common manifestation of stroke, a thalamic stroke can alter mental state but be easily missed if not specifically considered. Additional lab work-up should include a digoxin level and, since he is taking warfarin, a prothrombin time/ international normalized ratio (PT/INR). If the initial laboratory studies and head CT do not explain the altered mental state, magnetic resonance imaging (MRI) of the brain should be performed to further assess for stroke.

On physical examination in the ED, the patient was resting comfortably with eyes closed, and arousing to voice. He obeyed commands and participated in the examination. His Glasgow Coma Scale score was 13; temperature, $36.8^{\circ} \mathrm{C}$, heart rate, 80 beats per minute; respiratory rate, 16 breaths per minute; blood pressure, $90 / 57 \mathrm{~mm} \mathrm{Hg}$; and $100 \%$ peripheral capillary oxygen saturation while breathing ambient air. He appeared well developed. His heart rhythm was irregularly irregular, without murmurs, rubs, or gallops. Respiratory and abdominal examination findings were normal. The left BKA incision was well approximated, with no drainage, dehiscence, fluctuance, or erythema. On neurologic examination, the patient was intermittently oriented only to self. Pupils were equal, round, and reactive to light; extraocular movements were intact; face was symmetric; tongue was midline; sensation on face was equal bilaterally; and shoulder shrug was intact. Strength was 5/5 and symmetric in the elbow and hip and $5 / 5$ in the right knee and ankle (not tested on left because of BKA). Deep tendon reflexes were $3+$ and symmetrical at the biceps, brachioradialis, and triceps tendons and $3+$ in the right patellar and Achilles tendons. Sensation was intact and symmetrical in the upper and lower extremities. The patient's speech was slow and slurred, and his answers were unrelated to the questions being asked.

The patient's mental state is best described as lethargic. As he is only intermittently oriented, he meets the criteria for delirium. He is not obtunded or comatose, and his pupils are at least reactive, not pinpoint, so narcotic overdose is less likely. Thalamic stroke remains in the differential diagnosis; despite the seemingly symmetrical sensation examination, hemisensory deficits cannot be definitively ruled out given the patient's mental state. A rare entity such as carcinomatosis meningitis or another diffuse, infiltrative neoplastic process could be causing his condition. However, because focal deficits other than abnormal speech and diffuse hyperreflexia are absent, toxic, infectious, or metabolic causes are more likely than structural abnormalities. Still possible is a medication toxicity, such as ertapenem toxicity or, less likely, digoxin toxicity. In terms of infectious possibilities, urinary tract infection could certainly present in this fashion, especially if the patient had a somewhat low neurologic reserve at baseline, and hypotension could be secondary to sepsis. Encephalitis or meningitis remains in the differential diagnosis, though the patient appears nontoxic, and therefore a bacterial etiology is very unlikely.
The patient's hyperreflexia may be an important clue. Although the strength of his reflexes at baseline is unknown, seizures can cause transiently increased reflexes as well as a confused, lethargic mental state. Reflexes can also be increased by a drug overdose that has caused serotonin syndrome. Of the patient's medications, only ondansetron can cause this reaction. Hyperthyroidism can cause brisk reflexes and confusion, though more typically it causes agitated confusion. A thyroid-stimulating hormone level should be added to the initial laboratory panel.

A complete blood count revealed white blood cell count $11.86 \mathrm{~K} / \mathrm{uL}$ with neutrophilic predominance and immature granulocytes, hemoglobin $11.5 \mathrm{~g} / \mathrm{dL}$, and platelet count $323 \mathrm{~K} / \mathrm{uL}$. Serum sodium was $141 \mathrm{mEq} / \mathrm{L}$, potassium $4.2 \mathrm{mEq} / \mathrm{L}$, chloride $103 \mathrm{mEq} / \mathrm{L}$, bicarbonate $30 \mathrm{mEq} / \mathrm{L}$, creatinine $1.14 \mathrm{mg} / \mathrm{dL}$ (prior baseline of 0.8 $1.0 \mathrm{mg} / \mathrm{dL}$ ), blood urea nitrogen $26 \mathrm{mg} / \mathrm{dL}$, blood glucose $159 \mathrm{mg} / \mathrm{dL}$, and calcium $9.1 \mathrm{mg} / \mathrm{dL}$. His digoxin level was $1.3 \mathrm{ng} / \mathrm{mL}$ (reference range $0.5-1.9 \mathrm{mg} / \mathrm{mL}$ ) and troponin was undetectable. INR was 2.7 and partial thromboplastin time (PTT) 60 seconds. Vitamin $B_{12}$ level was $674 \mathrm{pg} / \mathrm{mL}$ (reference range $>180$ ). A urinalysis had $1+$ hyaline casts and was negative for nitrites, leukocyte esterase, blood, and bacteria. An ECG revealed atrial fibrillation with a ventricular rate of 80 beats per minute. A chest radiograph showed clear lung fields. A CT of the head without IV contrast had no evidence of an acute intracranial abnormality. In the ED, 1 liter of IV normal saline was given and blood pressure improved to $127 / 72 \mathrm{~mm} \mathrm{Hg}$.

The head CT does not show intracranial bleeding, and, though it is reassuring that INR is in the therapeutic range, ischemic stroke must remain in the differential diagnosis. Sepsis is less likely given that the criteria for systemic inflammatory response syndrome are not met, and hypotension was rapidly corrected with administration of IV fluids. Urinary tract infection was ruled out with the negative urinalysis. Subclinical seizures remain possible, as does medication-related or other toxicity. A medication overdose, intentional or otherwise, should also be considered.

The patient was admitted to the hospital. On reassessment by the inpatient team, he was oriented only to self, frequently falling asleep, and not recalling earlier conversations when aroused. His speech remained slurred and difficult to understand. Neurologic examination findings were unchanged since the ED examination. On additional cerebellar examination, he had dysmetria with finger-to-nose testing bilaterally and dysdiadochokinesia (impaired rapid alternating movements) of the left hand.

His handedness is not mentioned; the dysdiadochokinesia of the left hand may reflect the patient's being right-handed, or may signify a focal cerebellar lesion. The cerebellum is also implicated by the bilateral dysmetria. Persistent som- 
TABLE. Naranjo Adverse Drug Reaction Questionnaire

\begin{tabular}{lcc}
\hline Questions & Yes & No \\
\hline 1. Are there previous conclusive reports on this reaction? & +1 & 0 \\
\hline 2. Did the adverse events appear after the suspected drug was given? & +2 & -1 \\
\hline 3. Did the adverse reaction improve when the drug was discontinued or a specific antagonist was given? & +1 & 0 \\
\hline 4. Did the adverse reaction reappear when the drug was readministered? & +2 & -1 \\
\hline 5. Are there alternative causes that could have caused the reaction? & -1 & +2 \\
\hline 6. Did the reaction reappear when a placebo was given? & -1 & +1 \\
\hline 7. Was the drug detected in any body fluid in toxic concentrations? & +1 & 0 \\
\hline 8. Was the reaction more severe when the dose was increased, or less severe when the dose was decreased? & +1 & 0 \\
\hline 9. Did the patient have a similar reaction to the same or similar drugs in any previous exposure? & +1 & 0 \\
\hline 10. Was the adverse event confirmed by any objective evidence? & +1 & 0 \\
\hline
\end{tabular}

Score: $\geq 9$, definite; 5-8, probable; $1-4$, possible; $\leq 0$, doubtful.

nolence in the absence of CT findings suggests a metabolic or infectious process. Metabolic processes that can cause bilateral cerebellar ataxia and somnolence include overdose of a drug or medication. Use of alcohol or a medication such as phenytoin, valproic acid, or a benzodiazepine can cause the symptoms in this case, but was not reported by the family, and there was no documentation of it in the SNF records. Wernicke encephalopathy is rare and is not well supported by the patient's presentation but should be considered, as it can be easily treated with thiamine. Meningoencephalitis affecting the cerebellum remains possible, but infection is less likely. Both electroencephalogram and brain MRI should be performed, with a specific interest in possible cerebellar lesions. If the MRI is unremarkable, a lumbar puncture should be performed to assess opening pressure and investigate for infectious etiologies.

MRI of the brain showed age-related volume loss and nonspecific white matter disease without acute changes. Lack of a clear explanation for the neurologic findings led to suspicion of a medication side effect. Ertapenem was stopped on admission because it has been reported to rarely cause altered mental status. IV moxifloxacin was started for the osteomyelitis. Over the next 2 days, symptoms began resolving; within 24 hours of ertapenem discontinuation, the patient was awake, alert, and talkative. On examination, he remained dysarthric but was no longer dysmetric. Within 48 hours, the dysarthria was completely resolved, and he was returned to the SNF to complete a course of IV moxifloxacin.

\section{DISCUSSION}

Among elderly patients presenting to the $\mathrm{ED}$, altered mental status is a common complaint, accounting for $10 \%$ to $30 \%$ of visits. ${ }^{1}$ Medications are a common cause of altered mental status among the elderly and are responsible for $40 \%$ of delirium cases. ${ }^{1}$ The risk of adverse drug events (ADEs) rises with the number of medications prescribed. ${ }^{1-3}$ Among patients older than 60 years, the incidence of polypharmacy (defined as taking $>5$ prescription medications) increased from roughly $20 \%$ in 1999 to $40 \%$ in $2012.4,5$ The most common ADEs in the ambulatory setting (25\%) are central nervous system (CNS) symptoms, including dizziness, sleep disturbances, and mood changes. ${ }^{6}$ A medication effect should be suspected in any elderly patient presenting with altered mental state.

The present patient developed a constellation of neurologic symptoms after starting ertapenem, one of the carbapenem antibiotics, which is a class of medications that can cause CNS ADEs. Carbapenems are renally cleared, and adjustments must be made for acute or chronic changes in kidney function. Carbapenems are associated with increased risk of seizure; the incidence of seizure with ertapenem is $0.2 \%{ }^{7,8}$ Food and Drug Administration postmarketing reports have noted ertapenem can cause somnolence and dyskinesia, ${ }^{9}$ and several case reports have described ertapenem-associated CNS side effects, including psychosis and encephalopathy. ${ }^{10-13}$ Symptoms and examination findings can include confusion, disorientation, garbled speech, dysphagia, hallucinations, miosis, myoclonus, tremor, and agitation. ${ }^{10-13}$ Although reports of dysmetria and dysdiadochokinesia are lacking, suspicion of an ADE in this case was heightened by the timing of the exposure and the absence of alternative infectious, metabolic, and vascular explanations for bilateral cerebellar dysfunction.

The Naranjo Adverse Drug Reaction (ADR) scale may help clinicians differentiate ADEs from other etiologies of symptoms. It uses 10 weighted questions (Table) to estimate the probability that an adverse clinical event is caused by a drug reaction. ${ }^{14}$ The present case was assigned 1 point for prior reports of neurologic ADEs associated with ertapenem, 2 for the temporal association, 1 for resolution after medication withdrawal, 2 for lack of alternative causes, and 1 for objective evidence of neurologic dysfunction-for a total of 7 
points, indicating ertapenem was probably the cause of the patient's neurologic symptoms. Of 4 prior cases in which carbapenem toxicity was suspected and the Naranjo scale was used, 3 found a probable relationship, and the fourth a highly probable one. ${ }^{10,12}$ Confusion, disorientation, hallucinations, tangential thoughts, and garbled speech were reported in the 3 probable cases of ADEs. In the highly probable case, tangential thoughts, garbled speech, and miosis were noted on examination, and these findings returned after re-exposure to ertapenem. Of note, these ADEs occurred in patients with normal and abnormal renal function, and in middle-aged and elderly patients. ${ }^{10,11,13}$

Most medications have a long list of low-frequency and rarely reported adverse effects. The present case reminds clinicians to consider rare adverse effects, or variants of previously reported adverse effects, in a patient with unexplained symptoms. To estimate the probability that a drug is causing harm to a patient, using a validated tool such as the Naranjo scale helps answer the question, What are the chances?

\section{KEY TEACHING POINTS}

- Clinicians should include rare adverse effects of common medications in the differential diagnosis.

- The Naranjo score is a validated tool that can be used to systematically assess the probability of an adverse drug effect at the bedside.

- The presentation of ertapenem-associated neurotoxicity may include features of bilateral cerebellar dysfunction.

Disclosure: Nothing to report.

\section{References}

1. Inouye SK, Fearing MA, Marcantonio ER. Delirium. In: Halter JB, Ouslander JG, Tinetti ME, Studenski S, High KP, Asthana S, eds. Hazzard's Geriatric Medicine and Gerontology. 6th ed. New York, NY: McGraw-Hill; 2009.

2. Sarkar U, López A, Maselli JH, Gonzales R. Adverse drug events in U.S. adult ambulatory medical care. Health Serv Res. 2011;46(5):1517-1533.

3. Chrischilles E, Rubenstein L, Van Gilder R, Voelker M, Wright K, Wallace R. Risk factors for adverse drug events in older adults with mobility limitations in the community setting. J Am Geriatr Soc. 2007;55(1):29-34.

4. Kaufman DW, Kelly JP, Rosenberg L, Anderson TE, Mitchell AA. Recent patterns of medication use in the ambulatory adult population of the United States: the Slone survey. JAMA. 2002;287(3):337-344.

5. Kantor ED, Rehm CD, Haas JS, Chan AT, Giovannucci EL. Trends in prescription drug use among adults in the United States from 1999-2012. JAMA. 2015;314(17):1818-1831.

6. Thomsen LA, Winterstein AG, Søndergaard B, Haugbølle LS, Melander A. Systematic review of the incidence and characteristics of preventable adverse drug events in ambulatory care. Ann Pharmacother. 2007;41(9):1411-1426.

7. Zhanel GG, Wiebe R, Dilay L, et al. Comparative review of the carbapenems. Drugs. 2007;67(7):1027-1052.

8. Cannon JP, Lee TA, Clark NM, Setlak P, Grim SA. The risk of seizures among the carbapenems: a meta-analysis. J Antimicrob Chemother. 2014;69(8):2043-2055.

9. US Food and Drug Administration. Invanz (ertapenem) injection [safety information]. http://www.fda.gov/Safety/MedWatch/SafetyInformation/ucm196605.htm. Published July 2013. Accessed July 6, 2015.

10. Oo Y, Packham D, Yau W, Munckhof WJ. Ertapenem-associated psychosis and encephalopathy. Intern Med J. 2014;44(8):817-819.

11. Wen MJ, Sung CC, Chau T, Lin SH. Acute prolonged neurotoxicity associated with recommended doses of ertapenem in 2 patients with advanced renal failure. Clin Nephrol. 2013;80(6):474-478.

12. Duquaine S, Kitchell E, Tate T, Tannen RC, Wickremasinghe IM. Central nervous system toxicity associated with ertapenem use. Ann Pharmacother. 2011;45(1):e6.

13. Kong V, Beckert L, Awunor-Renner C. A case of beta lactam-induced visual hallucination. N Z Med J. 2009;122(1298):76-77.

14. Naranjo CA, Busto U, Sellers EM, et al. A method for estimating the probability of adverse drug reactions. Clin Pharmacol Ther. 1981;30(2):239-245. 